\title{
On the Approximate Minimization of Functionals*
}

\author{
By James W. Daniel
}

\begin{abstract}
This paper considers in general the problem of finding the minimum of a given functional $f(u)$ over a set $B$ by approximately minimizing a sequence of functionals $f_{n}\left(u_{n}\right)$ over a "discretized" set $B_{n}$; theorems are given proving the convergence of the approximating points $u_{n}$ in $B_{n}$ to the desired point $u$ in $B$. Applications are given to the Rayleigh-Ritz method, regularization, Chebyshev solution of differential equations, and the calculus of variations.
\end{abstract}

1. Introduction. Many theoretical and computational problems either arise or can be formulated as one of locating a minimizing point of some real-valued (nonlinear) functional over a certain set; such variational settings often lead to existence theorems as well as to computational methods for solving the problems in question. Computationally, however, one is generally forced to deal with discrete data in place of the original functional; it is therefore necessary to analyze the relationships between variational problems and their discretized analogues.

In [7, Section 4], we first studied under certain equicontinuity assumptions the question of approximately minimizing one functional by minimizing a sequence of nearby functionals. In this present note we state the problem generally, give some convergence theorems, and describe some particular examples.

2. Minimization over $W$-Compact Sets. Let $E$ be a normed linear space and let $f$ be a real-valued (nonlinear) functional on $E$. Let there be another notion of convergence (i.e., a topology) in $E$ in addition to norm-convergence, $W$-continuity, $W$-compactness, etc. For example, if $E$ is a reflexive Banach space, the $W$-topology might be the weak-topology. We wish to minimize $f$ over a $W$-compact set $B$.

Definition 2.1. A discretization for the functional $f$ on $E$ consists of a family of normed linear spaces $E_{n}$, a family of real-valued functionals $f_{n}$ on $E_{n}$, a family of mappings $p_{n}$ of $E_{n}$ into $E$, and a family of mappings $r_{n}$ of $E$ into $E_{n}$.

Definition 2.2. A discretization for $f$ on $E$ is consistent if

(1) $\lim _{n \rightarrow \infty} \sup f_{n}\left(r_{n} \hat{u}\right) \leqq f(\hat{u})$ if $\hat{u}$ minimizes $f$ over the set in question,

(2) $\lim _{n \rightarrow \infty} \sup \left[f\left(p_{n} u_{n}\right)-f_{n}\left(u_{n}\right)\right] \leqq 0$ for any sequence $u_{n} \in E_{n}$ such that $p_{n} u_{n}$ remains in the set $B$ over which we wish to minimize $f$.

Remark. Generally one would demonstrate that (1) is valid for $\hat{u}$ by proving its validity for all $u$ in $E$.

We now state the basic minimization problem over $B(\mathrm{MP} B)$ : Let $f$ be $W$-lower semicontinuous and bounded below on a $W$-compact set $B$; find $\hat{u} \in B$ such that $f(\hat{u}) \leqq f(u)$ for all $u \in B$.

Remark. At least one such $\hat{u}$ exists [17]. Recall that lower semicontinuity means $f(u) \leqq \lim \inf f\left(u_{n}\right)$ whenever $u_{n} W$-converges to $u$.

We wish to find $\hat{u}$ solving MPB by solving similar problems for $f_{n}$ on $E_{n}$. Thus

Received October 21, 1968.

* Prepared under Contract Number N00014-67-A-0128-0004 at the University of Wisconsin. Reproduction in whole or in part is permitted for any purpose of the United States Government. 
consider the basic approximating minimization problem over $B_{n}\left(\mathrm{MP} B_{n}\right)$ : Let $f_{n}$ be bounded below on sets $B_{n}$ satisfying $p_{n} B_{n} \subset B$ and $r_{n} B \subset B_{n}$; find $\hat{u}_{n} \in B_{n}$ such that $f_{n}\left(\hat{u}_{n}\right) \leqq f_{n}\left(u_{n}\right)+\epsilon_{n}$ for all $u_{n} \in B_{n}, \epsilon_{n}>0$ converging to zero.

We can now prove the following fundamental theorem on the approximate minimization of functionals.

Theorem 2.1. Let $f$ be $W$-lower semicontinuous and bounded below on a $W$-compact set $B$ and suppose $f_{n}, E_{n}, p_{n}, r_{n}$ gives a consistent discretization for $f$ on $E$ with sets $B_{n} \subset E_{n}$ satisfying $p_{n} B_{n} \subset B, r_{n} B \subset B_{n}$. Then there exist $\hat{u}$ and $\hat{u}_{n}$ solving MPB and $\mathrm{MP} B_{n}$. For any such solutions, $\lim _{n \rightarrow \infty} f\left(p_{n} \hat{u}_{n}\right)=\lim _{n \rightarrow \infty} f_{n}\left(\hat{u}_{n}\right)=f(\hat{u})$ and all W-limit points of $p_{u} \hat{u}_{n}$, at least one of which exists, solve MPB. If MPB has a unique solution, then $p_{n} \hat{u}_{n} W$-converges to it.

Proof. The existence of $\hat{u}$ was noted earlier; the existence of $\hat{u}_{n}$ is obvious. Since $\hat{u}$ solves $\mathrm{MP} B$, for all $n$ we have $f(\hat{u}) \leqq f\left(p_{n} \hat{u}_{n}\right) \equiv f_{n}\left(\hat{u}_{n}\right)+\eta_{n}$ where $\eta_{n} \equiv f\left(p_{n} \hat{u}_{n}\right)-$ $f_{n}\left(\hat{u}_{n}\right)$ satisfies $\lim _{n \rightarrow \infty} \sup \eta_{n} \leqq 0$ by part two of the consistency assumption. On the other hand, $\hat{u}_{n}$ solves MPB $B_{n}$ in the sense that $f_{n}\left(\hat{u}_{n}\right) \leqq \inf _{B_{n}} f_{n}\left(u_{n}\right)+\epsilon_{n}$; therefore we have $f(\hat{u}) \leqq f\left(p_{n} \hat{u}_{n}\right)=f_{n}\left(\hat{u}_{n}\right)+\eta_{n} \leqq \inf _{B_{n}} f_{n}\left(u_{n}\right)+\epsilon_{n}+\eta_{n} \leqq f_{n}\left(r_{n} \hat{u}\right)+\epsilon_{n}+\eta_{n}$, the latter inequality resulting from the fact that $r_{n} \hat{u} \in B_{n}$. By part one of the consistency assumption $\lim _{n \rightarrow \infty} \sup f_{n}\left(r_{n} \hat{u}\right) \leqq f(\hat{u})$; therefore by letting $n$ tend to infinity in the last string of inequalities we obtain $\lim _{n \rightarrow \infty} f\left(p_{n} \hat{u}_{n}\right)=\lim _{n \rightarrow \infty} f_{n}\left(\hat{u}_{n}\right)=f(\hat{u})$. Since $B$ is $W$-compact and $p_{n} \hat{u}_{n} \in B$, there exists at least one $W$-limit point of $p_{n} \hat{u}_{n}$ in $B$; let $u^{\prime}$ be any such point associated with a subsequence $p_{n_{j}} \hat{u}_{n_{j}}$. The $W$ lower semicontinuity of $f$ yields $f(\hat{u}) \leqq f\left(u^{\prime}\right) \leqq \lim _{n \rightarrow \infty} \inf f\left(p_{n_{j}} u_{n_{j}}\right)=f(\hat{u})$, so $u^{\prime}$ solves MPB; if the solution to MPB is unique, the sequence $p_{n} \hat{u}_{n}$ clearly is $W$ convergent to it. Q.E.D.

This theorem is a strengthening of Proposition 1, Section 4, in [7], which is concerned with collective compactness and thereby with approximate solutions of integral equations. Somewhat similar results, in essence directed toward Hammerstein equations, were obtained in unpublished notes by J.-P. Aubin and J. L. Lions [4]; their results, other than those giving explicit conditions on the Hammerstein operators which guarantee the satisfaction of the assumptions of our theorems, are contained in Theorem 2.1. The general ideas concerning discretization schemes are those of [2], [3], in which examples of $p_{n}$ and $r_{n}$ are given.

In practice it is often necessary to minimize a given functional over the entire space $E$; under further restrictions on the discretization scheme, we can handle this case also.

3. Minimization over $E$. Consider the following global minimization problem over $E(\mathrm{MP} E)$ : Let $f$ be $W$-lower semicontinuous and bounded below on $E$; find $\hat{u}$ such that $f(\hat{u}) \leqq f(u)$ for all $u \in E$.

Remark. A solution to MPE need not exist.

We approximate this problem by the following approximating global minimization problem over $E_{n}\left(\mathrm{MP} E_{n}\right)$ : Let $f_{n}$ be bounded below on $E_{n}$. Find $\hat{u}_{n}$ such that $f_{n}\left(\hat{u}_{n}\right) \leqq f_{n}\left(u_{n}\right)+\epsilon_{n}$ for all $u_{n} \in E_{n}$, where $\epsilon_{n}>0$ converges to zero.

Remark. $\mathrm{MPE}_{n}$ always has a solution.

We now wish to study situations in which solutions to MPE exist and can be obtained via solutions to $\mathrm{MPE}$. The type of condition that usually is imposed to 
guarantee the existence of a solution to $\mathrm{MPE}$ is a growth condition enabling us to restrict ourselves to bounded sets and then use results for that case. Therefore we now assume that solid spheres in $E$ are $W$-compact, i.e., for all real $R, S_{R}$ $\equiv\{u ;\|u\| \leqq R\}$ is $W$-compact.

Definition 3.1. The functional $f$ is said to satisfy a $T$-condition if there exists an $R>0$ and $u_{0}$ in $E$ with $\left\|u_{0}\right\| \leqq R$ such that $\|u\|>R$ implies $f(u)>f\left(u_{0}\right)$.

Remark. If there exists a real number $b$ and a monotone function $s(t)$ such that $\lim _{n \rightarrow \infty} s(t)=\infty$ and such that $f(u) \geqq b+s(\|u\|)$, then $f$ satisfies a $T$-condition.

Thus, for a functional satisfying a $T$-condition, problem MPE can be reduced to problem MPB over the $W$-compact set $B \equiv S_{R}$, for which we know a solution exists. It is possible, however, to have a consistent discretization for a functional $f$ satisfying a $T$-condition but such that the points $\hat{u}_{n}$ satisfy $\lim _{n \rightarrow \infty}\left\|p_{n} \hat{u}_{n}\right\|=\infty$; thus we need further conditions in order to solve $\mathrm{MP} E$ via $M P E_{n}$. What we need is a type of uniform growth condition on the functionals $f_{n}$ and a stability condition on the discretization.

Definition 3.2. A discretization for $f$ on $E$ satisfies a uniform growth condition if $\lim _{n \rightarrow \infty} \sup f_{n}\left(u_{n}\right)=\infty$ whenever $\lim _{n \rightarrow \infty} \sup \left\|u_{n}\right\|_{n}=\infty$.

Definition 3.3. A discretization for $f$ on $E$ is stable if there exists a constant $A$ such that $\left\|p_{n} u_{n}\right\| \leqq A\left\|u_{n}\right\|_{n}$ for all $u_{n} \in E_{n}$ for all $n$.

Now we can prove the following theorem on solving MPE via MPE $E_{n}$.

Theorem 3.1. Let solid spheres in $E$ be $W$-compact. Let the $W$-lower semicontinuous functional $f$ be bounded below on $E$ and satisfy a T-condition with $R=R_{0}$. Let the given discretization for $f$ on $E$ be stable and consistent (condition 2 must hold if $\left\|p_{n} u_{n}\right\|$ is bounded) and satisfy a uniform growth condition; suppose each $f_{n}$ is bounded below on $E_{n}$. Then solutions $\hat{u}$ and $\hat{u}_{n}$ exist solving $\mathrm{MPE}$ and $\mathrm{MP} E_{n}$ respectively. For any such solutions $\lim _{n \rightarrow \infty} f\left(p_{n} \hat{u}_{n}\right)=\lim _{n \rightarrow \infty} f_{n}\left(\hat{u}_{n}\right)=f(\hat{u})$, and all $W$-limit points of $p_{n} \hat{u}_{n}$, at least one of which exists, solve MPE. If the solution to MPE is unique, then $p_{n} \hat{u}_{n} W$-converges to it.

Proof. By our assumptions, $\hat{u}_{n}$ and $\hat{u}$ exist. Since $r_{n} \hat{u} \in E_{n}$, we have $f_{n}\left(\hat{u}_{n}\right) \leqq$ $f_{n}\left(r_{n} \hat{u}\right)$ and $\lim _{n \rightarrow \infty} \sup f_{n}\left(r_{n} \hat{u}\right) \leqq f(\hat{u})$ by the consistency assumption; therefore there exists a constant $C$ such that $f_{n}\left(\hat{u}_{n}\right) \leqq C$ for all $u$ and hence, because of the uniform growth condition, there exists a constant $D$ such that $\left\|\hat{u}_{n}\right\|_{n} \leqq D$. Therefore, by stability, $\left\|p_{n} \hat{u}_{n}\right\| \leqq A\left\|u_{n}\right\|_{n} \leqq A D$. Let $R=\max \left(R_{0}, A D\right)$. Let $B_{n}=\left\{u_{n} ;\left\|u_{n}\right\|_{n} \leqq\right.$ $D\}, B=S_{R}=\{u ;\|u\| \leqq R\}$. The theorem now follows by applying Theorem 2.1 with the sets $B_{n}$ and $B$ as defined above, recalling that $B$ is $W$-compact. Q.E.D.

It is quite straightforward to apply our two main theorems to generate results concerning, for example, the approximate solution of nonlinear operator equations (by looking at conditions guaranteeing that $\hat{u}$ is an interior point of $B$ or of $E$ and deducing that the derivative of $f$ must vanish there) and the approximate solution of nonlinear eigenvalue-eigenvector problems (by guaranteeing $\hat{u}$ to be on the boundary of $B$ for certain types of sets and applying the extended Lagrange multiplier theorem). Results of this type are contained in Sections 4 and 5 of [7]; we pursue this no further here. Instead we look briefly at a number of methods currently in use for solving certain types of problems to see how they fit into the above theory and how the theory indicates the necessary characteristics of the particular methods. 


\section{Examples.}

I. Rayleigh-Ritz. Perhaps the simplest and oldest example is that of the RayleighRitz procedure. Here we let $E$ be a Hilbert space with complete basis $\phi_{1}, \cdots, \phi_{n}, \cdots$ and for each $n$ let $E_{n}$ be the subspace of $E$ spanned by $\phi_{1}, \cdots, \phi_{n}$, with $p_{n} u_{n}=u_{n}$ considered as an element of $E$, and $r_{n} u=\sum_{i=1}^{n}\left\langle u, \boldsymbol{\phi}_{i}\right\rangle \boldsymbol{\phi}_{i}$ where $\langle u, v\rangle$ is the inner product on $E$; the inner product on $E_{n}$ is the one induced by $\langle\cdot, \cdot\rangle$ on $E$. Given a functional $f$, let $f_{n}=f$ for all $n$. Such a discretization is stable and, if $f$ is norm-continuous, it is consistent. A great deal of interest has arisen lately in the application of this method to the numerical solution of differential equations using various bases $\phi_{1}, \cdots$, that is, different finite-dimensional subspaces $E_{n}$. In particular, in [5] it is shown how very good error bounds can be computed; essentially this approach assumes that $f$ has a differentiable gradient $J=\nabla f$ with uniformly positive-definite derivative $J^{\prime}$. If $\hat{u}\left(\hat{u}_{n}\right)$ minimizes $f\left(f_{n}\right)$ on $E\left(E_{n}\right)$ then $J(\hat{u})=0$ and $\left\langle J\left(\hat{u}_{n}\right), \phi_{i}\right\rangle=0, i=1, \cdots, n$; hence $0=\left\langle J(\hat{u})-J\left(\hat{u}_{n}\right), \phi_{i}\right\rangle=$ $\left\langle J^{\prime}\left(\hat{u}_{n}+\vartheta\left(\hat{u}-\hat{u}_{n}\right)\right)\left(\hat{u}-\hat{u}_{n}\right), \phi_{i}\right\rangle$ which states that $\hat{u}_{n}$ is the projection under the inner product $[u, v] \equiv\left\langle J^{\prime}\left(\hat{u}_{n}+\vartheta\left(\hat{u}-\hat{u}_{n}\right)\right) u, v\right\rangle$ of $\hat{u}$ onto $E_{n}$. Thus $\left[\hat{u}-\hat{u}_{n}, \hat{u}-\hat{u}_{n}\right] \leqq\left[\hat{u}-u_{n}, \hat{u}-u_{n}\right]$ for all $u_{n} \in E_{n}$ which provides an error estimate. Details and extensions of this approach are contained in [5], [6].

One difficulty not mentioned in the above papers is that of minimizing $f_{n}$ over $E_{n}$; in this case $f_{n}$ is an integral of a complicated differential form, often nonlinear. In practice this is discretized by a quadrature sum so that we do not in fact have $f_{n}=f$. The difficulties therein created are treated in [9].

II. Regularization. Often one seeks not just any arbitrary minimizing point to a functional but one which, in some sense, is smoothest or most regular. For example, let $f$ and $g$ be weakly lower semicontinuous functionals on a reflexive Banach space $E$, and let $g$ be nonnegative. Let $E_{n}=E, p_{n}=r_{n}=$ the identity, $f_{n}=f+a_{n} g$ where $a_{n}>0$ converges to zero. Let $B$ be a weakly compact set in $E$ with $f$ bounded below on $B$. The consistency condition (1) becomes $\lim _{n \rightarrow \infty} \sup \left[f(\hat{u})+a_{n} g(\hat{u})\right] \leqq$ $f(u)$ which clearly is satisfied. Condition (2) becomes $\lim _{n \rightarrow \infty}$ sup $-a_{n} g\left(u_{n}\right) \leqq 0$ which follows from the nonnegativity of $a_{n}$ and $g$. For future reference we note that $f\left(\hat{u}_{n}\right)+a_{n} g\left(\hat{u}_{n}\right) \leqq f(\hat{u})+a_{n} g(\hat{u}) \leqq f\left(\hat{u}_{n}\right)+a_{n} g(\hat{u})$, implying $g\left(\hat{u}_{n}\right) \leqq g(\hat{u})$. It follows then from Theorem 2.1 that any weak limit point $u^{\prime}$ of $\left\{u_{n}\right\}$ minimizes $f$ over $B$; moreover, since $g\left(u^{\prime}\right) \leqq \lim _{n \rightarrow \infty} \inf g\left(\hat{u}_{n}\right) \leqq g(\hat{u}), g\left(u^{\prime}\right)$ is the minimum value of $g$ over the set of minimizing points of $f$ in $B$. One says that the minimizing point has been regularized by $g$ [11], [16]; if $B$ is convex and $g$ is uniformly convex, then $u^{\prime}$ is in fact a norm limit point and moreover if $f$ is quasi-convex [12], the entire sequence $\left\{u_{n}\right\}$ converges in norm to $u^{\prime}[11]$.

III. Chebyshev Solution of Differential Equations. Suppose one seeks to solve $A u=b$ where $A$ is a uniformly elliptic linear differential operator in two variables over a bounded domain $D$, under the condition $u=0$ on $\Gamma$, the boundary of $D$, assumed to be sufficiently smooth; more general types of equations may also be treated by the method to be presented. A numerical method of recent popularity [10], [14] given a sequence of functions $\left\{\phi_{i}\right\}$ satisfying the boundary data, consists in choosing numbers $a_{n, 1}, \cdots, a_{n, n}$ to minimize $\max _{1 \leqq j \leqq M}\left|\left[A\left(\sum_{i=1}^{n} a_{n, i} \phi_{i}\right)\right]\left(x_{j}\right)-b\left(x_{j}\right)\right|$ where the $M$ points $\left\{x_{j}\right\}$ form a "grid" over $D$. Strictly for convenience we take $M=4 n$ and suppose that the grid is such that any point in $D$ is at a distance of 
at most $h_{n}$ from a grid-point $x_{j}$. We wish to find conditions under which $\hat{u}_{n} \equiv$ $\sum_{i=1}^{n} a_{n, i} \phi_{i}$ will converge, in some sense, to the solution $\hat{u}$ of our problem.

Since we seek to minimize a supremum norm, the norm must be defined; so let $E=\{u ; u=0$ on $\Gamma$, all partial derivatives of $u$ through second order are continuous on $\bar{D}=D \cup \Gamma\} ;$ for $u \in E$, let $\|u\|=\|u\|_{\infty}=\max _{\bar{D}}|u(x)|$. Let $f(u)=\|A u-b\|_{\infty}$, where we now need to assume that $b$ is bounded on $\bar{D}$. Let $E_{n}$ be that subset of $E$ spanned by the functions $\phi_{1}, \cdots, \phi_{n}$, assumed to lie in $E$; let $p_{n}$ be the identity mapping, and $r_{n}$ be at the moment undefined. Define

$$
f_{n}\left(u_{n}\right)=\left\|A u_{n}-b\right\|_{4 n, \infty}=\max _{1 \leqq i \leqq 4 n}\left|\left[A u_{n}\right]\left(x_{i}\right)-b\left(x_{i}\right)\right|
$$

we now seek conditions for consistency. Consider (2) of our Definition 2.2. $f\left(p_{n} u_{n}\right)-f_{n}\left(u_{n}\right)=\left\|A u_{n}-b\right\|_{\infty}-\left\|A u_{n}-b\right\|_{4 n, \infty}$. Since this quantity is always nonnegative, the requirement $\lim _{n \rightarrow \infty} \sup \left[f\left(p_{n} u_{n}\right)-f_{n}\left(u_{n}\right)\right] \leqq 0$ in fact demands convergence; in order to compare suprema over discrete and continuous sets, we need to know something about the growth of the functions $A u_{n}-b$ between gridpoints. Hence we now need to assume that $A \phi_{i}$ satisfies a Lipschitz condition with Lipschitz constant $\lambda_{i}$ (this restricts $A$ somewhat also) and that $b$ satisfies one with a constant $\lambda_{0}$. From this it follows that $\left|f\left(p_{n} u_{n}\right)-f_{n}\left(u_{n}\right)\right| \leqq h_{n} \sum_{i=0}^{n}\left|a_{n, i}\right|\left|\lambda_{i}\right|$, where $a_{n, 0}=1$. Thus we need next a growth condition on $\sum_{i=0}^{n}\left|a_{n, i}\right|\left|\lambda_{i}\right|$. For example, the conditions that (i) there exists a constant $C$ such that $\sum_{i=0}^{n}\left|a_{n, i}\right| \leqq C$ for all $n$, and (ii) $h_{n} \Lambda_{n}$ tends to zero, where $\Lambda_{n}=\max _{1 \leqq i \leqq n} \lambda_{i}$, would be sufficient; in practice the $\Lambda_{n}$ do in fact become large, while the restriction on the $a_{n, i}$ is easy to implement. In essence, the above restrictions are defining $B_{n}$, i.e., $B_{n}=\left\{u_{n} ; \sum_{i=0}^{n}\left|a_{n, i}\right| \leqq C\right\}$.

Next consider condition (1) of Definition 2.2, where $r_{n}$ is to be defined. We require $\lim _{n \rightarrow \infty} \sup f_{n}\left(r_{n} u\right) \leqq f(u)$. Now $f_{n}\left(r_{n} u\right) \leqq f\left(r_{n} u\right)$, so we need only require that $\lim _{n \rightarrow \infty} \sup f\left(r_{n} u\right) \leqq f(u)$; this is certainly true if $r_{n} u$ is an approximation method in which $A r_{n} u$ converges uniformly to $A u$, for example, if $r_{n} u$ and all its partial derivatives through second-order converge uniformly to those for $u$. We remark that it is necessary to have $r_{n} u$ in $B_{n}$.

Under the above conditions, it follows from Theorem 2.1 and its proof that $\lim _{n \rightarrow \infty} f_{n}\left(\hat{u}_{n}\right)=\lim _{n \rightarrow \infty} f\left(p_{n} \hat{u}_{n}\right)=f(\hat{u})=0$, where $\hat{u}$ solves $A \hat{u}=b$ and lies in $E$; the conditions on $W$-compactness and $W$-lower semicontinuity are needed only to prove convergence for $p_{n} u_{n}$, a problem easily handled differently here. We know that $\left\|A \hat{u}_{n}-b\right\|_{\infty}=f\left(p_{n} u_{n}\right)$ converges to zero. By a simple use of the maximum principle [13], we deduce $\left\|u_{n}-\hat{u}\right\|_{\infty} \leqq\left\|A \hat{u}_{n}-b\right\|_{\infty}\|w\|_{\infty}$ where $w$ solves $A w=-1$ in $D$, $w=0$ on $\Gamma$; therefore $\hat{u}_{n}$ converges uniformly to the solution $\hat{u}$.

The application of the theory in Section 2 to this problem indicates the type of approach necessary to prove convergence for this numerical method. One requires: (1) smooth functions $\phi_{i}$ with Lipschitz constants $\lambda_{i}$ for $A \phi_{i}$ that do not grow too rapidly, (2) results from approximation theory that state that if one approximates functions $b$ by combinations of functions $A \phi_{i}$, the sums $\sum_{i=1}^{n}\left|a_{n, i}\right|$ remain bounded, and (3) functions $b$ can be approximated by functions $A \phi_{i}$. The requirements (1) and (3) here are probably less difficult; generalized Bessel inequality results like (2), however, are not known to this author for general cases. While numerical work with this method proceeds, theoretical results of the type suggested by our theorem should be sought. 
IV. Calculus of Variations. Consider the problem of minimizing the functional $f(u)=\int_{0}^{1} g(t, u, \dot{u}) d t, u(0)=u(1)=0$, where $\dot{u}=d u / d t$. The following simple case of a general numerical method has been suggested [8]: minimize

$f_{n}\left(u_{n}\right)=\sum_{i=1}^{n} h_{i} g\left(t_{i-1}, u_{n, i-1}, \frac{u_{n, i}-u_{n, i-1}}{h_{i}}\right), \quad u_{n, 0}=u_{n, n}=0, \quad h_{i}=t_{i}-t_{i-1}$,

where the minimization is over the set of values of $u_{n, 1}, \cdots, u_{n, n-1}$; this method can be fit neatly into the theory of Theorem 2.1. In [8], under the assumption that there exist unique minimizing points for $f$ (in $C^{1}[0,1]$ ) and $f_{n}$ satisfying the "spike" condition $\left|\left(\hat{u}_{n, i}-\hat{u}_{n, i-1}\right) / h_{i}\right| \leqq A$ for some constant $A$ independent of $n$, it was purportedly proved that $p_{n} \hat{u}_{n}$, the piecewise-linear interpolation to $\hat{u}_{n}$, converges uniformly to $\hat{u}$; because the authors inadvertently left out an assumption guaranteeing a lower semicontinuity property for the functional $f$, the proof is in fact incorrect. However, as we shall show below by use of Theorem 2.1, the usual assumptions guaranteeing a unique minimizing point for $f$, in conjunction with an assumption guaranteeing the satisfaction of a type of spike condition, yield a convergence proof.

For convenience let us take $h_{i}=h=1 / n$ for all $i$. For a fixed $p>1$, let $E=$ $\left\{u ; u(0)=u(1)=0, u\right.$ is absolutely continuous on $\left.[0,1], \dot{u} \in L_{p}[0,1]\right\} ;$ for $u \in E$, let

$$
\|u\|=\|\dot{u}\|_{p}=\left\{\int_{0}^{1}|\dot{u}(t)|^{p} d t\right\}^{1 / p} .
$$

For each $n$, let $E_{n}$ be $(n-1)$-dimensional Euclidean space, where $u_{n} \in E_{n}$ has the norm

$$
\left\|u_{n}\right\|_{n}=\left\{h \sum_{i=1}^{n}\left[\left|\frac{u_{n, i}-u_{n, i-1}}{h}\right|\right]^{p}\right\}^{1 / p}
$$

where $u_{n, 0}=u_{n, n}=0$ by definition. Let $p_{n}$ be the mapping defined by piecewiselinear joining of the values $u_{n, i}$ at the points $t_{i}=i h$, thus $p_{n} u_{n} \in E$. Define the mapping $r_{n}$ via $\left(r_{n} u\right)_{i}=u\left(t_{i}\right), i=1, \cdots, n-1$. Define $W$-convergence as follows: A sequence $u^{(n)}$ in $E W$-converges to $u$ in $E$ if $\int_{0}^{1}\left(\dot{u}^{(n)}-\dot{u}\right) v d t$ converges to zero for every function $v(t) \in L_{q}(0,1), 1 / p+1 / q=1$.

Proposition 4.1. Solid spheres in $E$ are $W$-compact. If $u^{(n)} W$-converges to $u$, then $u^{(n)}$ converges to $u$ uniformly, i.e., in the supremum norm.

Proof. If we take any sequence $u^{(n)}$ in $E$ with $\left\|u^{(n)}\right\|=\left\|\dot{u}^{(n)}\right\|_{p} \leqq R$, then, by Alaoglu's Theorem [15, p. 228], there exists a subsequence $u^{\left(n_{j}\right)}$ and a function $s$ in $L_{p}$ such that for all $v$ in $L_{q}, \int_{0}^{t}\left(\dot{u}^{\left(n_{j}\right)}-s\right) v d t$ tends to zero. Let $u(t)=\int_{0}^{t} s(t) d t$; it follows that $u \in E$ and $u^{\left(n_{j}\right)} W$-converges to $u$. For the second part of the proof, we note first that $\left\|u^{(n)}\right\|$ is bounded, by the uniform-boundedness principle [15, p. 202]. Thus

$$
\begin{aligned}
\left|u^{(n)}\left(t_{1}\right)-u^{(n)}\left(t_{2}\right)\right| & \leqq \int_{t_{1}}^{t_{2}}\left|\dot{u}^{(n)}\right| d t \leqq\left[\int_{t_{1}}^{t_{2}}\left|\dot{u}^{(n)}\right|^{p} d t\right]^{1 / p}\left|t_{2}-t_{1}\right|^{1 / q} \\
& \leqq\left\|u^{(n)}\right\|\left|t_{2}-t_{1}\right|^{1 / q}
\end{aligned}
$$

which implies that the sequence $u^{(n)}$ is uniformly bounded and equicontinuous. If $u^{(n)}$ does not converge uniformly to $u$, then there exists $\epsilon>0$ and a sequence $t_{j}$ such 
that $\left|u^{\left(n_{j}\right)}\left(t_{j}\right)-u\left(t_{j}\right)\right| \geqq \epsilon$; applying Ascoli's theorem [15, p. 276] to the bounded, equicontinuous sequence $u^{\left(n_{j}\right)}$ yields a contradiction. Q.E.D.

We now make the standard type of assumption in the calculus of variations in order to guarantee the existence of a minimizing point for $f(u)$.

Assumptions. (i) $g(t, u, w)$ is jointly continuous in its variables for $0 \leqq t \leqq 1$ and $-\infty \leqq u, w \leqq \infty$. (ii) There exist constants $a, b$ with $b>0$ such that $g(t, u, w) \geqq$ $a+b|w|^{p}$ for all $t$ in $[0,1]$, $u$ finite. (iii) $g$ is differentiably convex in $w$, i.e., $g\left(t, u, w_{1}\right)$ $-g\left(t, u, w_{2}\right) \geqq\left(w_{1}-w_{2}\right) g_{w}\left(t, u, w_{2}\right)$.

Proposition 4.2. The functional $f$ is $W$-lower semicontinuous on $E$, bounded below, and satisfies a T-condition.

Proof. For the last two statements, note that $f(u)=\int_{0}^{1} g(t, u, \dot{u}) d t$ $\geqq \int_{0}^{1}\left[a+b|\dot{u}|^{p}\right] d t=a+b\|u\|^{p}$. The proof of the $W$-lower semicontinuity is straightforward using the convexity of $g$; details may be found in [1, p. 137-139]. Q.E.D.

Proposition 4.3. The discretization scheme defined above is stable and satisfies a uniform growth condition.

Proof.

$$
\begin{aligned}
\left\|p_{n} u_{n}\right\|^{p} & =\int_{0}^{1}\left|\left(p_{n} u_{n}\right)^{\cdot}\right|^{p} d t=\sum_{i=1}^{n} \int_{t_{i-1}}^{t_{i}}\left|\left(p_{n} u_{n}\right)^{\cdot}\right|^{p} d t \\
& =h \sum_{i=1}^{n}\left[\frac{\left.\left|u_{n, i}-u_{n, i-1}\right|\right]^{p}}{h}=\left\|u_{n}\right\|_{n}^{p} .\right. \\
f_{n}\left(u_{n}\right) & =h \sum_{i=1}^{n} g\left(t_{i-1}, u_{n, i-1}, \frac{u_{n, i}-u_{n, i-1}}{h}\right) \geqq h \sum_{i=1}^{n}\left[a+b\left|\frac{u_{n, i}-u_{n, i-1}}{h}\right|^{p}\right] \\
& =a+b\left\|u_{n}\right\|_{n}^{p} . \quad \text { Q.E.D. }
\end{aligned}
$$

The only remaining ingredient for application of Theorem 3.1 is the consistency; in [8], the spike condition was needed for this. In our case, we must make the following assumptions.

Assumptions. (iv) Some solution $\hat{u}$ minimizing $f(u)$ lies in $C^{1}[0,1]$, i.e., $\hat{u}^{\cdot}$ is continuous. (v) There exist constants $c$ and $d$ and a continuous function $s(t, v)$ such that $\left|g\left(t_{1}, v_{1}, z\right)-g\left(t_{2}, v_{2}, z\right)\right| \leqq\left(c+d|z|^{p}\right)\left|s\left(t_{1}, v_{1}\right)-s\left(t_{2}, v_{2}\right)\right|$ where $t_{1}, t_{2}$ are arbitrary points in $[0,1]$ and $v_{1}, v_{2}, z$ are arbitrary real numbers.

Remark. If $g(t, u, w) \equiv w^{2} / 2+r(t, u)$, then (v) is satisfied with $s \equiv r$. If $g(t, u, w)$ $\equiv l(w) m(t, u)$ with $|l(w)| \leqq c+d|w|^{p}$, then (v) is satisfied with $r \equiv m$; many actual problems are of the above types.

Proposition 4.4. The discretization is consistent.

Proof. For condition (1), we prove $\lim _{n \rightarrow \infty}\left|f_{n}\left(r_{n} \hat{u}\right)-f(\hat{u})\right|=0$. Since, by assumption, $\hat{u}$ is in $C^{1}[0,1]$, given $\epsilon$, for sufficiently large $n,\left|\hat{u}\left(t_{i-1}\right)-\hat{u}(t)\right|<\epsilon$ and $\left|\hat{u}^{\cdot}(t)-\left(\hat{u}_{i}-\hat{u}_{i-1}\right) / h\right|<\epsilon$ for $t_{i-1} \leqq t \leqq t_{i}$. For convenience, we write merely $u$ for $\hat{u}$.

$$
\left|f(u)-f_{n}\left(r_{n} u\right)\right| \leqq \sum_{i=1}^{n} \int_{t_{i-1}}^{t_{i}}\left|g(t, u, \dot{u})-g\left(t_{i-1}, u_{i-1}, \frac{u_{i}-u_{i-1}}{h}\right)\right| d t .
$$

But, by uniform continuity of $g$, given $\delta>0$ there exists $\epsilon>0$ and then $N$ such that $n>N$ implies $\left|f(u)-f_{n}\left(r_{n} u\right)\right| \leqq \sum_{i=1}^{n} \int_{t_{i-1}}^{t_{i}} \delta d t=\delta$. Since $\delta>0$ was arbitrary, con- 
dition (1) is proved. For condition (2), we show that $\lim _{n \rightarrow \infty}\left|f\left(p_{n} u_{n}\right)-f_{n}\left(u_{n}\right)\right|=0$ if $\left\|p_{n} u_{n}\right\|$ is bounded.

$$
\begin{array}{r}
\left|f\left(p_{n} u_{n}\right)-f_{n}\left(u_{n}\right)\right| \leqq h \sum_{i=1}^{n} \int_{0}^{1} \mid g\left(t_{i-1}+\alpha h,(1-\alpha) u_{n, i-1}+\alpha u_{n, i}, \frac{u_{n, i}-u_{n, i-1}}{h}\right) \\
\quad-g\left(t_{i-1}, u_{n, i-1}, \frac{u_{n, i}-u_{n, i-1}}{h}\right) \mid d \alpha \\
\leqq h \sum_{i=1}^{n} \int_{0}^{1}\left(c+d\left|\frac{u_{n, i}-u_{n, i-1}}{h}\right|^{p}\right) \\
\cdot\left|s\left(t_{i-1}+\alpha h,(1-\alpha) u_{n, i-1}+\alpha u_{n, i}\right)-s\left(t_{i-1}, u_{n, i-1}\right)\right| d \alpha .
\end{array}
$$

Now $\left\|u_{n}\right\|_{n}=\left\|p_{n} u_{n}\right\|$ is bounded, $\left|u_{n, i}\right|$ is bounded, and $\left|u_{n, i}-u_{n, i-1}\right| \leqq h^{1-1 / p}\left\|u_{n}\right\|_{n}$. Thus, using the uniform continuity of $s(t, u)$, given $\epsilon>0$ there exists $N$ such that $n>N$ implies

$$
\left|f\left(p_{n} u_{n}\right)-f_{n}\left(u_{n}\right)\right| \leqq h \sum_{i=1}^{n} \int_{0}^{1}\left(c+d\left|\frac{u_{n, i}-u_{n, i-1}}{h}\right|^{p}\right) \epsilon d \alpha=\epsilon\left[c+d\left\|u_{n}\right\|_{n}^{p}\right]
$$

since $\epsilon>0$ was arbitrary, condition (2) follows. Q.E.D.

We now can state the following theorem which follows immediately from Theorem 3.1 and the above propositions.

Theorem 4.1. Let Assumptions (i) - (v) be valid and let the discretization method described above be used. Then all $W$-limit points of $p_{n} \hat{u}_{n}$ (at least one of which exists) minimize $f$. If the solution $\hat{u}$ is unique, then in particular $p_{n} \hat{u}_{n}$ converges uniformly to $\hat{u}$ and the derivatives converge $L_{p}$-weakly.

Computer Sciences Department

The University of Wisconsin

Madison, Wisconsin 53706

1. N. I. Ahiezer, The Calculus of Variations, GITTL, Moscow, 1955; English transl., Blaisdell, Waltham, Mass., 1962. MR 17, 861; MR 25 \#5414.

2. J.-P. Aubin, "Approximation des espaces de distribution et des opérateurs différentiels," Bull. Soc. Math. France, Mémoire No. 12, 1967.

3. J.-P. Aubin, "Evaluation des erreurs de troncature des approximations des espaces de Sobolev," J. Math. Anal. Appl., v. 21, 1968, pp. 356-368. MR 36 \#6923.

4. J.-P. Aubin \& J. L. Lions, Unpublished Notes on Minimization, Private Communication.

5. P. G. Ciarlet, M. H. Schultz \& R. S. VARGA, "Numerical methods of high-order accuracy for nonlinear boundary value problems. I: One dimensional problem," Numer. Math., v. 9, 1967, pp. 394-430. MR 36 \#4813.

6. P. G. Ciarlet, M. H. Schultz \& R. S. VARga, "Numerical methods of high order accuracy for nonlinear boundary value problems. Monotone operators." (To appear.)

7. J. W. DANIEL, "Collectively compact sets of gradient mappings," Proc. Nederl. Akad. Wetensch., v. 71, 1968, pp. 270-279.

8. D. Greenspan, "On approximating extremals of functionals. II," Internat. J. Engrg. Sci., v. 5, 1967, pp. 571-588. MR $36 \# 3508$.

9. R. J. Herbold, Constant Quadrature Schemes for the Numerical Solution of Bonndary Value Problems by Variational Techniques, Ph.D. Thesis, Case Western Reserve Univ., Cleveland, Ohio, 1968.

10. W. KRABs, Einige Methoden zur Lösung des diskreten linearen Tschebyscheff-Problems, Dissertation, University of Hamburg, 1963.

11. E. S. LEvitin \& B. T. PolyaK, "Convergence of minimizing sequences in problems on the relative extremum," Dokl. Akad. Nauk SSSR, v. 168, 1966, pp. 997-1000 = Soviet Math. Dokl., v. 7, 1966, pp. 764-767. MR 33 \#7166. 
12. O. L. Mangasarian, "Pseudo-convex functions," J. SIAM Ser. A Control, v. 3, 1965, pp. 281-290. MR 32 \#9062.

13. M. H. Protter \& H. F. Weinberger, Maximum Principles in Differential Equations, Prentice-Hall, Englewood Cliffs, N. J., 1967. MR 36 \#2935.

14. J. B. Rosen, A pproximate Solution and Error Bounds for Quasi-Linear Elliptic Boundary Value Problems, Univ. of Wisconsin, Computer Sciences Technical Report No. 30, 1968.

15. A. E. TAYLOR, Introduction to Functional Analysis, Wiley, New York and Chapman \& Hall, London, 1958. MR $20 \# 5411$.

16. A. N. Truonov, "Regularisation methods for optimal control problems," Dokl. Akad. Nauk SSSR, v. 162, 1965, pp. 763-765= Soviet Math. Dokl., v. 6, 1965, pp. 761-763. MR 31 \#3274. 17. M. M. VAINBERG, Variational Methods for the Study of Nonlinear Operators, GITTL, Moscow, 1956; English transl., Holden-Day, San Francisco, Calif., 1964, p. 78. MR 19, 567; MR $31 \# 638$. 